\title{
Predictors of the response to nivolumab immunotherapy in the second or subsequent lines for metastatic non-small cell lung cancers
}

\author{
SORIN SĂFTESCU ${ }^{1}$, ȘERBAN NEGRU ${ }^{1}$, SIMONA VOLOVĂȚ ${ }^{2}$, DOREL POPOVICI $^{1}$, \\ VLAD CHERCOTA $^{3}$, SIMONA STANCA ${ }^{4}$, HOREA FEIER ${ }^{5}$, DANIEL MALITA ${ }^{6}$, \\ RADU DRAGOMIR ${ }^{7}$ and CONSTANTIN VOLOVĂȚ ${ }^{2}$
}

\author{
${ }^{1}$ Department of Oncology, 'Victor Babeș’ University of Medicine and Pharmacy, 300041 Timisoara; \\ ${ }^{2}$ Department of Oncology, 'Gr. T. Popa' University of Medicine and Pharmacy, 700115 Iasi; \\ ${ }^{3}$ Department of Ophthalmology, 'Victor Babeș' University of Medicine and Pharmacy, 300041 Timisoara; \\ ${ }^{4}$ Department of Pediatrics, 'Carol Davila' University of Medicine and Pharmacy, 020021 Bucharest; \\ Departments of ${ }^{5}$ Cardiovascular Surgery, ${ }^{6}$ Radiology and Medical Imaging, ${ }^{7}$ Obstetrics and Gynecology, \\ 'Victor Babeș' University of Medicine and Pharmacy, 300041 Timisoara, Romania
}

Received October 30, 2020; Accepted December 1, 2020

DOI: $10.3892 / \mathrm{etm} .2021 .10037$

\begin{abstract}
Checkpoint inhibitors represent the first therapeutic class to replace chemotherapy lines for the treatment of metastatic non-small cell lung cancer (NSCLC), due to improved overall survival and tolerability. Nivolumab, a fully human anti-programmed cell death-1 immunoglobulin G4 monoclonal antibody, is the first immune checkpoint inhibitor approved by the US Food and Drug Administration in 2014 for cases of metastatic melanoma and in 2015 for cases of squamous cell lung cancer and kidney cell cancer. The present study aimed to identify predictive markers (favorable or unfavorable) for time to treatment discontinuation using nivolumab in the second or subsequent line of therapy of metastatic NSCLC cases. Analysis of a group of 78 NSCLC patients treated with nivolumab allowed the identification of negative predictive markers, related to the presence of metastases (adrenal in men under 65 years, liver, brain and the number of metastatic sites) and the hematological profile (neutrophilia at the initiation of treatment and lymphocyte variation at 6 weeks of treatment).
\end{abstract}

Correspondence to: Dr Şerban Negru, Department of Oncology, 'Victor Babeș' University of Medicine and Pharmacy, 2 Eftimie Murgu Square, 300041 Timişoara, Romania

E-mail: snegru@yahoo.com

Dr Simona Stanca, Department of Pediatrics, 'Carol Davila' University of Medicine and Pharmacy, 37 Dionisie Lupu Street, 020021 Bucharest, Romania

E-mail: simonastanca@yahoo.com

Key words: immunotherapy, NSCLC, non-small cell lung cancer, metastases, predictive, lymphocytes, adrenal, nivolumab

\section{Introduction}

One of the best documented mechanisms of immune evasion of cancer cells is inactivation of the immune response via programmed cell death protein 1 (PD-1)/programmed death ligand 1 (PD-L1) (1).

PD-1 is a membrane immunoglobulin present on the surface of $\mathrm{T}$ and pro-B lymphocytes, with a role in silencing the immune response by suppressing (following PD-L1 binding) $\mathrm{T}$ cell proliferation and activation. Nivolumab is an Ig4 monoclonal antibody that blocks PD-1/PD-L1 signaling by specific binding to PD-1; it disinhibits the antigen-dependent antitumor immune response. The use of nivolumab was approved by the US Food and Drug Administration in 2014 for cases of metastatic melanoma and in 2015 for cases of non-small cell lung cancer (NSCLC) and kidney cell cancer (2-4).

The activity of stimulating $\mathrm{T}$ cell reactivity by nivolumab is conditioned by the presence of antigenic stimuli for $\mathrm{T}$ cell receptors and starts from a dose of $1.5 \mathrm{ng} / \mathrm{ml}$ (5). Compared to chemotherapy, the wide therapeutic range of anti-PD-1 molecules has allowed the escalation of nivolumab doses, although the therapeutic efficacy also manifests at dosages of one tenth of those used in current practice (6).

To date, no maximum tolerated dose for nivolumab has been identified and toxicity profiles are similar over the dose range of $0.1-10 \mathrm{mg} / \mathrm{kg} \mathrm{q} 2 \mathrm{w}$, although a dose-effect relationship is manifested for NSCLC up to a dose of $3 \mathrm{mg} / \mathrm{kg}$ q2w (7). However, recent studies have shown that dose reduction (20 and $100 \mathrm{mg}$, fixed doses every 3 weeks, respectively) does not affect progression-free survival (PFS) or overall survival (OS) compared to $3 \mathrm{mg} / \mathrm{kg} \mathrm{q} 2 \mathrm{w}$ in patients with NSCLC (8).

The clearance of nivolumab does not depend on the type of tumor; it is increased in combination with ipilimumab and tends to decrease inversely proportional to body mass 
index (BMI) and albuminemia (9). In the case of NSCLC, one of the negative predictors for the response to immunotherapy (IT) is sarcopenia, one of the manifestations of cachexia (10). It is important to note that albumin deficiency may induce apparent hypocalcemia (11).

In the same context, low creatinine levels are associated with sarcopenia and cachexia and indicate a mediocre response to immunotherapy.

It is interesting to note that the serum level of nivolumab at 14,45 and 60 days after the beginning of treatment correlates with the plasma level of 25-hydroxycholecalciferol (12).

\section{Patients and methods}

The present study was approved by the Oncohelp Clinic Ethics Committee; all 78 patients included in the study voluntarily agreed to participate and provided written consent. We screened 692 admission charts/medical files (from January 1, 2019 to August 31,2020) and created a MySQL database with anthropometric (sex, age, height, BMI), imagistic (metastases topography), hematological (red and white blood cell count), biochemical [creatinine, calcium, alanine aminotransferase (ALT), aspartate aminotransferase (AST)] and therapeutic (the use of opioids) variables.

The statistical analysis of the association between the overall time on treatment with the anthropometric, imagistic, hematological, biochemical and therapeutic variables was performed using the Cox Proportional Hazards Survival Regression (CPHSR) test available at https://statpages.info/prophaz.html) and included both past and ongoing immunotherapy cases.

\section{Results}

There were no statistically significant differences found between the age and duration of immunotherapy in our study group, analysed by gender ( $28.2 \%$ women) (Table I).

In terms of age group distribution, we found no significant differences between males and females (Table II).

Analysis of anthropometric variables showed a statistically significant difference between height of the males and females, but not body mass index (BMI) (Table III).

Most of the patients included in the study exhibited a good biological status, all with Eastern Cooperative Oncology Group Performance Score (ECOG PS) $<3$ at the time of immunotherapy initiation (Table IV).

In contrast with the female patients, more male patients (76.9 vs. 55\%) showed various degrees of anemia (grade 1 and 2, $\mathrm{P}=0.067$ ) (Table V). On average, the hemoglobin $(\mathrm{Hb})$ level did not change significantly at 6 weeks upon therapy (the average change was $+0.09 \mathrm{~g} / \mathrm{dl}$ ) (Table VI). However, we noted wide individual variations [standard deviation $(\mathrm{SD})=1.30 \mathrm{~g} / \mathrm{dl}$ for $\mathrm{Hb}$ variation]. Of note, there were no statistically significant differences between $\mathrm{Hb}$ levels at initiation and the fourth cycle of immunotherapy ( $\mathrm{P}=0,6891$, Mann Whitney test).

Almost $26 \%$ of the patients showed an abnormal number of lymphocytes at baseline, while in the case of neutrophils, the percentage of anomalies was much lower (12.5\%). Similarly to the $\mathrm{Hb}$ variable, there were minimal global variations (on average $+0.06 \times 10^{9} / 1$ ) for absolute lymphocyte count (ALC) (Fig. 1) and absolute neutrophil count (ANC) (on average $+0.16 \times 10^{9} / 1$ ) at six weeks of immunotherapy, with wide individual variations $\left(\mathrm{SD}=0.55 \times 10^{9} / 1\right.$ for $\mathrm{ALC}$ variation and $\mathrm{SD}=4.06$ for $\mathrm{ANC}$ variation). Neutropenic cases were absent at 6 weeks and neutrophilia was present in $7.5 \%$ of cases $\left(\right.$ maximum value $\left.=39 \times 10^{9} / 1\right)$. Of note, there were few cases of status reversals (e.g. neutrophilia turned to normal range neutrophils).

Leukocyte count and platelet (PLT) count showed significant differences in regards to sex distribution at initiation (Table VII).

Creatinine, total calcium, ALT and AST showed no significant sex differences at initiation (Table VIII). There was a slight (statistically not significant) decrease in both creatinine (5.4\%) and calcium (1.7\%) levels during the first six weeks of therapy.

Next, we evaluated the overall number and the topography of metastases and found statistically significant differences in males vs. females, with female predilection towards brain and lung metastases (Table IX). In regard to brain metastases, the frequency in patients $<60$ years was significantly higher than in patients $\geq 60$ years $(P=0.00032)$. In addition, liver metastases were more frequent in patients $<60$ years of age $(\mathrm{P}=0.048)$. Moreover, there was a clear predilection of both the 40-49 and 50-59 age groups for brain metastasis (Table X). Interestingly, in our cohort, more males than females had no metastasis among evaluated sites (brain, lungs, liver, adrenal and bones), while females tend to be overrepresented in the 2 metastatic site subgroup (Table XI).

Evaluation of the immunotherapy status at 20 months showed that $15.7 \%$ of the patients underwent a single administration of nivolumab and the treatment was discontinued immediately. A total of $14.1 \%$ of the patients were treated for at least 2 cycles, but for less than 2 months and discontinued. A total of $21.8 \%$ of patients underwent at least 6 months of treatment (and from these patients, $14.1 \%$ are still in treatment) (Fig. 2).

The results of our CPHSR analysis are summarized [risk ratios (RR), 95\% CI intervals, P-values] (Table XII), with a focus on initiation and at six weeks of immunotherapy time points (Fig. 3).

\section{Discussion}

Following international incidence data on lung cancer (13), our cohort was formed mostly by men (71.8\%); there were no significant differences found between male and female patients in terms of age and age group distribution (yet to mention the 40-49 group, where females were predominant, $\mathrm{P}=0.075)$. There were no statistically significant differences between the body mass indices (BMIs) of the male and female patients, although males were significantly taller $(\mathrm{P}<0.00001)$. The vast majority of our patients $(88.5 \%)$ were in good biological status, with ECOG PS below 2; there were no differences between ECOG PS of the male and female patients $(\mathrm{P}=0.741)$.

The analysis of hematologic parameters showed a surprisingly higher percentage of anemia in men vs. women (which could be explained by wider interval for grade I anemia in men), while leukocyte and platelet counts were significantly higher in male vs. female patients. There were no significant 
Table I. Age and duration of treatment by sex.

\begin{tabular}{|c|c|c|c|c|}
\hline Variables & Males and females & Males & Females & P-value \\
\hline Age (years), average \pm SD & $63.6 \pm 8.4$ & $64.3 \pm 7.5$ & $62.0 \pm 10.3$ & 0.509 \\
\hline Duration of immunotherapy (days), average \pm SD & $130.5 \pm 140.7$ & $129.8 \pm 144.0$ & $132.9 \pm 132.3$ & 0.689 \\
\hline
\end{tabular}

$\mathrm{SD}$, standard deviation.

Table II. Age group distribution of the patients by sex.

\begin{tabular}{|c|c|c|c|c|c|}
\hline Age group, years & $40-49$ & $50-59$ & $60-69$ & $70-79$ & $\geq 80$ \\
\hline Males and females (\%) & 9.0 & 20.5 & 43.6 & 24.4 & 2.5 \\
\hline Females $(\%)$ & 18.2 & 18.2 & 40.9 & 18.2 & 4.5 \\
\hline Males (\%) & 5.4 & 21.4 & 44.6 & 26.8 & 1.8 \\
\hline z-test (males vs. females) & 0.075 & 0.748 & 0.764 & 0.423 & 0.496 \\
\hline
\end{tabular}

Table III. Height and BMI of patients by sex.

\begin{tabular}{lcc}
\hline Sex distribution & Height $(\mathrm{cm})$ & BMI $\left(\mathrm{kg} / \mathrm{m}^{2}\right)$ \\
\hline Males and females & $169.7 \pm 9.2$ & $25.2 \pm 5.9$ \\
Females & $174.0 \pm 6.4$ & $26.2 \pm 6.0$ \\
Males & $159.3 \pm 6.0$ & $24.6 \pm 5.9$ \\
P-value (males vs females) & $<0.00001$ & 0.453 \\
\hline
\end{tabular}

BMI, body mass index.

differences between male and female biochemical parameters at baseline.

The mean variation in hemoglobinemia $(\mathrm{Hb})$ revealed that a significant recovery from hematopoiesis from previous toxic chemotherapeutic effect only occurred in some of the patients.

The prevalence of lung and brain metastases was double and more than double, respectively, among female patients; differences reached statistical significance for brain metastases $(\mathrm{P}=0.0104)$ and were close to the limit for lung metastases $(\mathrm{P}=0.0523)$, while the male population showed a 5-time higher prevalence for adrenal metastases $(\mathrm{P}=0.0394)$. Most women enrolled $(72.7 \%)$ had 1 or 2 metastatic sites, as compared to $51.8 \%$ in the male subgroup.

CPHSR analysis indicated that each additional baseline metastatic site (aggregated data for brain, lung, liver, adrenal, bone topography) increased the patient risk of treatment exit by $56 \%(\mathrm{P}=0.0035)$. Hepatic metastasis at baseline translated into a risk ratio $(\mathrm{RR})$ of $2.67(\mathrm{P}=0.0097)$, an important negative predictive value.

Baseline adrenal metastasis was a significant negative predictor only in the $<65$-year old male subgroup, for which a high $3.52 \mathrm{RR}(\mathrm{P}=0.0257)$ was calculated; this was in full agreement with published data showing a significant decrease in median overall survival (OS) in the presence of adrenal
Table IV. Patient distribution by ECOG and sex

\begin{tabular}{lccc}
\hline & \multicolumn{3}{c}{ ECOG PS } \\
\cline { 2 - 4 } Sex distribution & 0 & 1 & 2 \\
\hline Males and females (\%) & 38.5 & 50.0 & 11.5 \\
Females (\%) & 39.3 & 50.0 & 10.7 \\
Males (\%) & 36.4 & 50.0 & 13.6 \\
z-test (males vs. females) & 0.81 & 1.00 & 0.72 \\
\hline
\end{tabular}

ECOG PS, Eastern Cooperative Oncology Group Performance Score.

metastases (14). Of note, in our cohort, the adrenal metastasis prevalence was almost double in the 40-49/50-59 age groups in comparison with the other age groups.

As expected, cerebral metastasis has a negative impact on the overall time of treatment $(\mathrm{R} R=1.93, \mathrm{P}=0.044)$. Crinò et al showed a $39 \%$ disease control rate with a median OS of 8.6 months in NSCLC patients under therapy with nivolumab (15).

A baseline neutrophil count over $8 \times 10^{9} / 1$ and an ALC variation of $+1 \times 10^{9} / 1$ at six weeks on therapy were both negative predictive factors, with comparable $\mathrm{RR}=2.39(\mathrm{P}=0.027)$ and 2.52 ( $\mathrm{P}=0.0394)$, respectively. Of note, baseline ANC per se was found to be a much weaker negative predictor $(R R=1.10$, $\mathrm{P}=0.0406$ ).

Our data concerning ALC variation as a negative predictor may come as a surprise, since previously published data describe a positive correlation of ALC (at baseline and at 6 weeks on treatment) with OS upon nivolumab therapy (16). While Karantanos et al described static data, our approach emphasized a novel, more dynamic parameter: The absolute change in ALC between baseline and 6-week time point. Why a positive ALC variation at 6 weeks of therapy exerts a negative effect on overall time on treatment remains to be explored on much wider cohorts of patients. 
Table V. Hematological parameters at immunotherapy initiation.

\begin{tabular}{|c|c|c|c|c|}
\hline Parameters & Males and females (\%) & Males (\%) & Females $(\%)$ & P-value (males vs. females) \\
\hline \multicolumn{5}{|l|}{ Hemoglobin $(\mathrm{Hb})$} \\
\hline Grade 2 anemia & 15.3 & 15.4 & 15.0 & 0.97 \\
\hline Grade 1 anemia & 55.6 & 61.5 & 40.0 & 0.10 \\
\hline Normal Hb level & 29.1 & 23.1 & 40.9 & 0.13 \\
\hline \multicolumn{5}{|l|}{$\operatorname{ALC}\left(\times 10^{9} / 1\right)$} \\
\hline$<1$ & 25.0 & 25.0 & 25.0 & 1 \\
\hline $1-4.8$ & 73.6 & 73.1 & 75.0 & 0.87 \\
\hline$\geq 4.8$ & 1.2 & 1.9 & 0.0 & 0.52 \\
\hline \multicolumn{5}{|l|}{$\mathrm{ANC}\left(\mathrm{x} 10^{9} / 1\right)$} \\
\hline$<2$ & 1.4 & 0 & 5.0 & 0.11 \\
\hline $2-9.9$ & 87.5 & 86.5 & 90.0 & 0.69 \\
\hline$\geq 10$ & 11.1 & 13.5 & 5.0 & 0.30 \\
\hline
\end{tabular}

ALC, absolute lymphocyte count; ANC, absolute neutrophil count. Grade 3 anemia <8 g/dl; Grade 2 anemia: 8-9.9 g/dl; Grade 1 anemia: 10-11.2 for female patients, 10-13.6 for male patients. Normal hemoglobin value: $\geq 13.7$ for male, $\geq 11.3 \mathrm{~g} / \mathrm{dl}$ for female.

Table VI. Percent distribution of cases by hemoglobin at 6 weeks.

\begin{tabular}{|c|c|c|c|c|}
\hline & Males and females (\%) & Males (\%) & Females $(\%)$ & P-value (males vs. females) \\
\hline \multicolumn{5}{|l|}{ Hemoglobin (Hb) } \\
\hline Grade 3 Anemia & 1.9 & 2.6 & 0 & 0.45 \\
\hline Grade 2 anemia & 9.4 & 7.9 & 13.3 & 0.47 \\
\hline Grade 1 anemia & 66 & 71.1 & 53.3 & 0.14 \\
\hline Normal Hb level & 22.6 & 18.4 & 33.3 & 0.16 \\
\hline
\end{tabular}

Table VII. Other hematological parameters at immunotherapy initiation.

\begin{tabular}{lcccc}
\hline Parameters & $\begin{array}{c}\text { Males and females } \\
\text { (average } \pm \text { SD) }\end{array}$ & $\begin{array}{c}\text { Males } \\
\text { (average } \pm \text { SD) }\end{array}$ & $\begin{array}{c}\text { Females } \\
\text { (average } \pm \text { SD) }\end{array}$ & $\begin{array}{c}\text { P-value } \\
\text { (males vs. females) }\end{array}$ \\
\hline MCV (fL) & $91.7 \pm 7.2$ & $91.6 \pm 6.8$ & $91.5 \pm 8.0$ & 0.180 \\
Leukocyte count $\left(\times 10^{9} / 1\right)$ & $9.1 \pm 4.0$ & $9.9 \pm 4.3$ & $7.1 \pm 2.1$ & 0.0214 \\
PLT count $\left(\times 10^{9} / 1\right)$ & $313.9 \pm 123.3$ & $332.5 \pm 126.0$ & $265.4 \pm 100.9$ & 0.020 \\
\hline
\end{tabular}

SD, standard deviation; MCV, mean corpuscular volume; PLT, platelet.

Table VIII. Biochemical parameters at immunotherapy initiation.

\begin{tabular}{|c|c|c|c|c|}
\hline Parameters & $\begin{array}{l}\text { Males and females } \\
\quad(\text { mean } \pm \mathrm{SD})\end{array}$ & $\begin{array}{c}\text { Males } \\
(\text { mean } \pm \mathrm{SD})\end{array}$ & $\begin{array}{c}\text { Females } \\
(\text { mean } \pm \mathrm{SD})\end{array}$ & $\begin{array}{c}\text { P-value } \\
\text { (males vs.females) }\end{array}$ \\
\hline Creatinine (mg/dl) & $0.91 \pm 0.44$ & $0.92 \pm 0.45$ & $0.90 \pm 0.42$ & 0.872 \\
\hline Calcium (total) (mg/dl) & $9.63 \pm 0.89$ & $9.64 \pm 1.0$ & $9.6 \pm 0.5$ & 0.441 \\
\hline ALT (IU/l) & $21.6 \pm 29.6$ & $22.1 \pm 33.3$ & $20.6 \pm 17.5$ & 0.880 \\
\hline AST (IU/l) & $27.5 \pm 31.4$ & $28.8 \pm 36.7$ & $24.3 \pm 8.3$ & 0.342 \\
\hline
\end{tabular}

SD, standard deviation; ALT, alanine aminotransferase; AST, aspartate aminotransferase. 
Table IX. Sex distribution of metastases at immunotherapy initiation.

\begin{tabular}{lccccc}
\hline & \multicolumn{5}{c}{ Metastases } \\
\cline { 2 - 5 } Sex distribution & Brain $(\%)$ & Lung $(\%)$ & Liver $(\%)$ & Adrenal $(\%)$ & Bone (\%) \\
\hline Males + females & 21.8 & 25.6 & 14.1 & 19.2 & 17.9 \\
Females & 40.9 & 40.9 & 13.6 & 4.5 & 22.7 \\
Males & 14.3 & 19.6 & 14.3 & 25 & 16.1 \\
z-test (males vs. females) & 0.0104 & 0.0523 & 0.9442 & 0.0384 & 0.490 \\
\hline
\end{tabular}

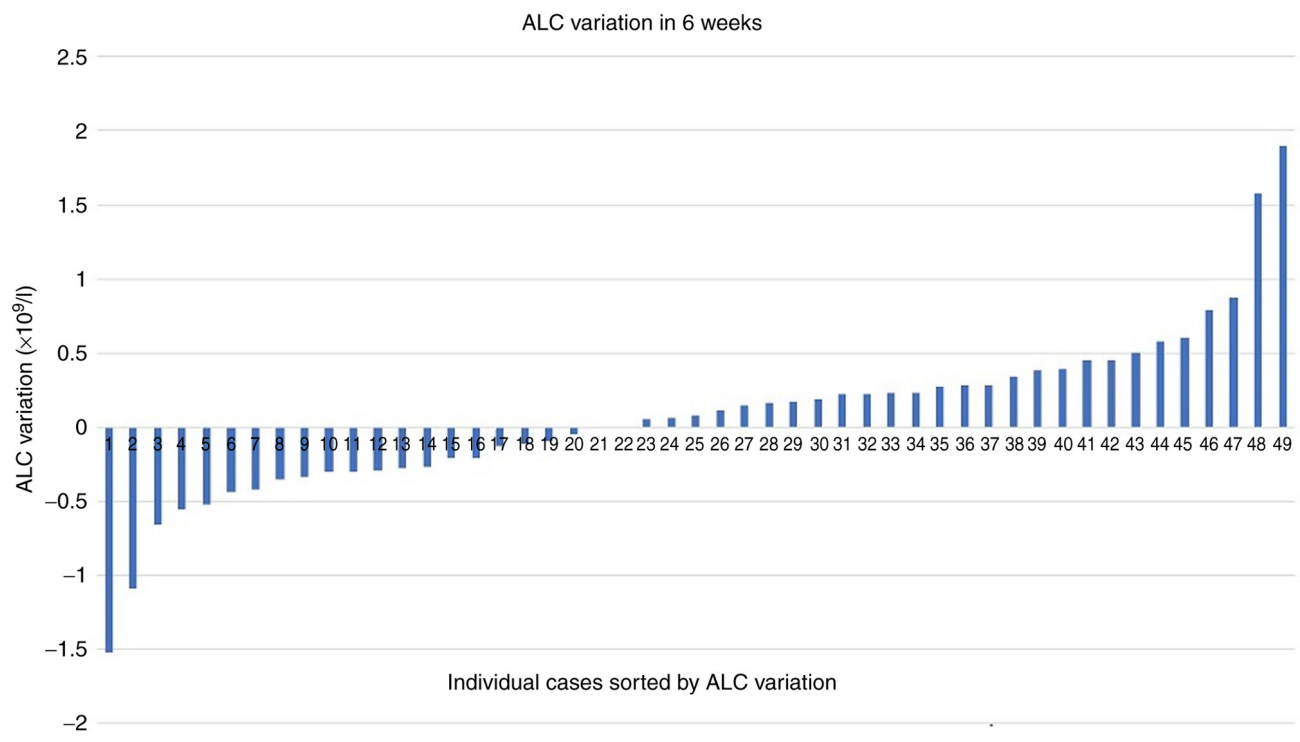

Figure 1. Individual variations in ALC at 6 weeks of treatment. ALC, absolute lymphocyte count.

Table X. Age distribution of metastases at baseline.

\begin{tabular}{lrrrrc}
\hline & \multicolumn{5}{c}{ Metastases } \\
\cline { 2 - 6 } Age distribution & Brain & Lung & Liver & Adrenal & Bone \\
\hline 40-49 years (\%) & 57 & 29 & 14 & 29 & 29 \\
50-59 years (\%) & 44 & 25 & 31 & 31 & 19 \\
60-69 years (\%) & 15 & 26 & 9 & 15 & 26 \\
70-79 years (\%) & 5 & 16 & 11 & 16 & 10 \\
$\geq 80$ years $(\%)$ & 0 & $100^{\text {a }}$ & 0 & 0 & 0 \\
\hline
\end{tabular}

anly two cases.

Table XI. Overall sex distribution in regards to metastatic site count at baseline.

\begin{tabular}{lcccccc}
\hline & \multicolumn{5}{c}{ Metastatic site number } \\
\cline { 2 - 7 } Sex distribution & $0^{\mathrm{a}}$ & 1 & 2 & 3 & 4 & 5 \\
\hline Males and females & 35.8 & 37.1 & 20.5 & 5.1 & 1.2 & 0 \\
Females (\%) & 22.7 & 40.9 & 31.8 & 4.5 & 0 & 0 \\
Males (\%) & 41.1 & 35.7 & 16.1 & 5.4 & 1.8 & 0 \\
z-test (males vs. females) & 0.13 & 0.67 & 0.12 & 0.87 & 0.53 & - \\
\hline
\end{tabular}

${ }^{\mathrm{a}}$ Other metastatic sites are present.

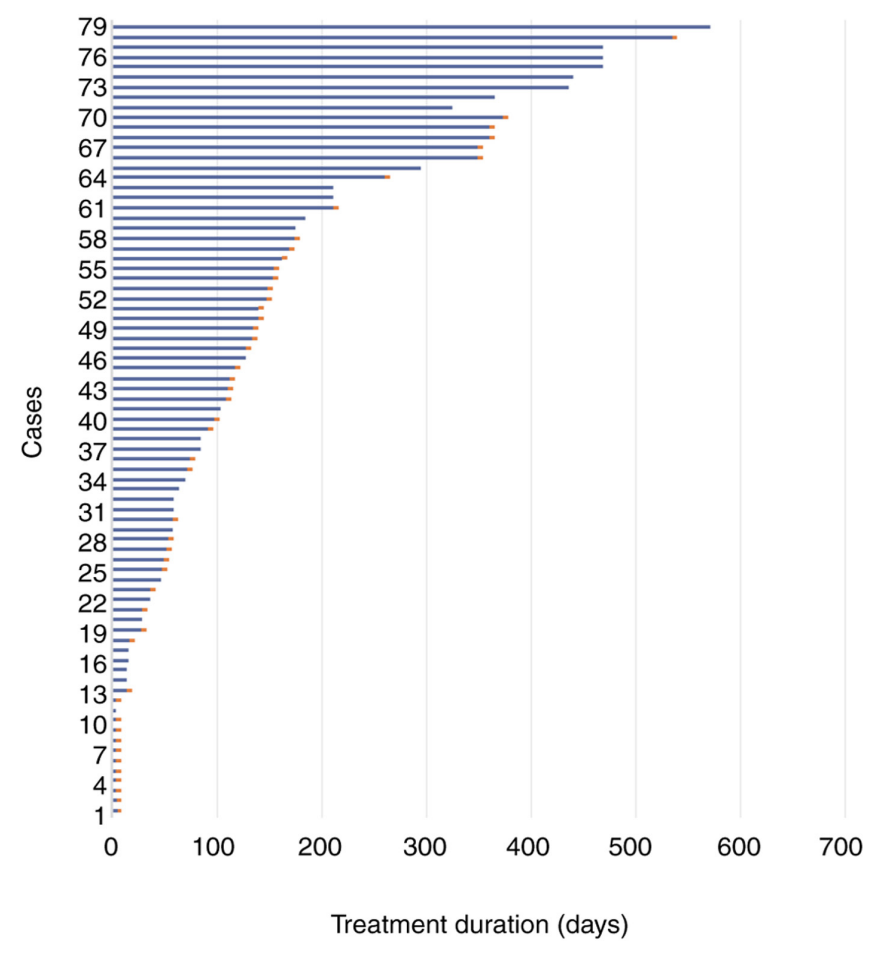

IT duration (days)

Figure 2. Nivolumab immunotherapy treatment duration and actual status. Ended treatments indicated in orange. 
Table XII. Parameters at initiation and 6 weeks of immunotherapy as analyzed by Cox proportional hazards survival regression.

\begin{tabular}{|c|c|c|c|c|c|c|}
\hline \multirow[b]{2}{*}{ Parameter } & \multicolumn{3}{|c|}{ Baseline parameters } & \multicolumn{3}{|c|}{$\begin{array}{c}\text { Parameter variation } \\
\text { after } 6 \text { weeks of nivolumab treatment }\end{array}$} \\
\hline & $\mathrm{RR}(95 \% \mathrm{CI})$ & P-value & No. of cases & $\mathrm{RR}(95 \% \mathrm{CI})$ & $\mathrm{P}$-value & No. of cases \\
\hline $\begin{array}{l}\text { Number of organs with metastases } \\
\text { (BRA+PUL+HEP+SR+OSS) } \\
\text { (values from } 0 \text { to } 5 \text {, average } 1.01 \text { ) }\end{array}$ & $\begin{array}{c}1.5569 \\
(1.156-2.095)\end{array}$ & 0.0035 & 76 & $\begin{array}{l}\text { NA } \\
\text { NA }\end{array}$ & - & - \\
\hline $\begin{array}{l}\text { Hepatic metastases present } \\
(0 / 1 \text {, average } 0.14)\end{array}$ & $\begin{array}{c}2.6651 \\
(1.268-5.599)\end{array}$ & 0.0097 & 76 & $\begin{array}{l}\text { NA } \\
\text { NA }\end{array}$ & - & \\
\hline $\begin{array}{l}\text { Adrenal metastases present, males } \\
<65 \text { years }(0 / 1 \text {, average } 0.24)\end{array}$ & $\begin{array}{c}3.5232 \\
(1.165-10.629)\end{array}$ & 0.0257 & 25 & $\begin{array}{l}\text { NA } \\
\text { NA }\end{array}$ & - & \\
\hline ANC $>8\left(\times 10^{9} / 1\right)(0 / 1$, average 0.18$)$ & $\begin{array}{c}2.3863 \\
(1.100-5.176)\end{array}$ & 0.0277 & 72 & $\begin{array}{c}2.2495 \\
(0.972-5.201)\end{array}$ & 0.0580 & 53 \\
\hline $\begin{array}{l}\operatorname{ALC}\left(\times 10^{9} / 1\right) \\
\text { (average 1.61) }\end{array}$ & $\begin{array}{c}0.9261 \\
(0.61-1.41)\end{array}$ & 0.7202 & 72 & $\begin{array}{c}2.5236 \\
(1.046-6.087)\end{array}$ & 0.0394 & 49 \\
\hline ANC (x109/1) (average 6.37) & $\begin{array}{c}1.1003 \\
(1.004-1.205)\end{array}$ & 0.0406 & 72 & $\begin{array}{c}1.0169 \\
(0.99-1.22)\end{array}$ & 0.0569 & 49 \\
\hline $\begin{array}{l}\text { Brain metastases present at initiation } \\
(0 / 1 \text {, average } 0.22)\end{array}$ & $\begin{array}{c}1.9332 \\
(1.017-3.671)\end{array}$ & 0.0440 & 76 & $\begin{array}{l}\text { NA } \\
\text { NA }\end{array}$ & - & - \\
\hline $\begin{array}{l}\text { Lung metastases present at initiation } \\
(0 / 1 \text {, average } 0.26)\end{array}$ & $\begin{array}{c}1.7943 \\
(0.98-3.26)\end{array}$ & 0.0562 & 76 & $\begin{array}{l}\text { NA } \\
\text { NA }\end{array}$ & - & - \\
\hline $\begin{array}{l}\text { Leukocyte count }\left( \pm \times 10^{9} / 1\right) \\
\text { (average } 9.13)\end{array}$ & $\begin{array}{c}1.066 \\
(0.996-1.140)\end{array}$ & 0.0637 & 72 & $\begin{array}{c}1.0877 \\
(0.89-1.31)\end{array}$ & 0.3920 & 49 \\
\hline Calcium total $<9 \mathrm{mg} / \mathrm{dl}$ at initiation & $\begin{array}{c}2.1237 \\
(0.80-5.58)\end{array}$ & 0.1267 & 67 & $\begin{array}{l}\text { NA } \\
\text { NA }\end{array}$ & - & - \\
\hline Male sex & $\begin{array}{c}1.6421 \\
(0.81-3.30)\end{array}$ & 0.1647 & 76 & $\begin{array}{c}1.6421 \\
(0.81-3.30)\end{array}$ & 0.1647 & 76 \\
\hline AST IU/l at initiation & $\begin{array}{c}1.0052 \\
(0.99-1.01)\end{array}$ & 0.21 & 70 & $\begin{array}{l}\text { NA } \\
\text { NA }\end{array}$ & - & - \\
\hline $\mathrm{Hb}$ at initiation $(\mathrm{g} / \mathrm{dl})$ & $\begin{array}{c}1.0108 \\
(0.85-1.21)\end{array}$ & 0.9053 & 72 & $\begin{array}{c}0.803 \\
(0.56-1.14)\end{array}$ & 0.2212 & 49 \\
\hline ALT IU/1 at initiation & $\begin{array}{c}1.0053 \\
(0.996-1.014)\end{array}$ & 0.2447 & 70 & $\begin{array}{l}\text { NA } \\
\text { NA }\end{array}$ & - & - \\
\hline Opioid usage at initiation & $\begin{array}{c}1.604 \\
(0.62-4.09)\end{array}$ & 0.3233 & 76 & $\begin{array}{c}1.1406 \\
(0.267-4.873)\end{array}$ & 0.8591 & 53 \\
\hline Adrenal metastases present at initiation & $\begin{array}{c}1.4187 \\
(0.69-2.88)\end{array}$ & 0.3332 & 76 & $\begin{array}{l}\text { NA } \\
\text { NA }\end{array}$ & - & - \\
\hline MCV (fL) at initiation & $\begin{array}{c}1.0165 \\
(0.97-1.05)\end{array}$ & 0.3864 & 72 & $\begin{array}{c}1.0036 \\
(0.94-1.06)\end{array}$ & 0.906 & 49 \\
\hline PLT count $\left(\times 10^{9} / 1\right)$ at initiation & $\begin{array}{c}1.001 \\
(0.998-1.003)\end{array}$ & 0.4456 & 72 & $\begin{array}{c}1.0032 \\
(0.998-1.008)\end{array}$ & 0.2157 & 49 \\
\hline Creatinine $(\mathrm{mg} / \mathrm{dl})$ at initiation & $\begin{array}{c}0.7559 \\
(0.36-1.55]\end{array}$ & 0.4473 & 71 & $\begin{array}{c}0.6269 \\
(0.08-4.54)\end{array}$ & 0.644 & 49 \\
\hline Height, male patients $(\mathrm{cm})$ & $\begin{array}{c}0.9874 \\
(0.93-1.04)\end{array}$ & 0.6353 & 52 & $\begin{array}{c}0.9874 \\
(0.93-1.04)\end{array}$ & 0.6353 & 52 \\
\hline Bone metastases present at initiation & $\begin{array}{c}0.848 \\
(0.37-1.90)\end{array}$ & 0.6892 & 76 & $\begin{array}{l}\text { NA } \\
\text { NA }\end{array}$ & - & - \\
\hline ECOG PS (0-4) at initiation & $\begin{array}{c}0.9133 \\
(0.58-1.42)\end{array}$ & 0.6895 & 76 & $\begin{array}{l}\text { NA } \\
\text { NA }\end{array}$ & - & - \\
\hline Age (years) & $\begin{array}{c}0.994 \\
(0.95-1.03)\end{array}$ & 0.7360 & 76 & $\begin{array}{c}0.994 \\
(0.95-1.03)\end{array}$ & 0.7360 & 76 \\
\hline Calcium total at initiation (mg/dl) & $\begin{array}{c}1.0713 \\
(0.71-1.62)\end{array}$ & 0.7445 & 67 & $\begin{array}{l}\text { NA } \\
\text { NA }\end{array}$ & & \\
\hline
\end{tabular}


Table XII. Continued.

Parameter variation

Baseline parameters

\begin{tabular}{|c|c|c|c|c|c|c|}
\hline \multirow[b]{2}{*}{ Parameter } & & \\
\hline & RR $(95 \%$ CI $)$ & P-value & No. of cases & RR $(95 \%$ CI $)$ & $\mathrm{P}$-value & No. of cases \\
\hline BMI at initiation $\left(\mathrm{kg} / \mathrm{m}^{2}\right)$ & $\begin{array}{c}0.9917 \\
(0.93-1.04)\end{array}$ & 0.7621 & 70 & $\begin{array}{l}\text { NA } \\
\text { NA }\end{array}$ & & \\
\hline
\end{tabular}

RR, risk ratio; CI, confidence interval; BRA, brain; PUL, pulmonary; HEP, hepatic; ADR, adrenal; OSS, osseous; ANC, absolute neutrophil count; ALC, absolute leucocyte count; ALT, alanine aminotransferase; AST, aspartate aminotransferase; Hb, hemoglobin; MCV, mean corpuscular volume; PLT, platelets; ECOG PS, Eastern Cooperative Oncology Group performance status; BMI, body mass index; NA, not applicable.

dentified risk ratios for $\mathrm{p}<0.05$ parameters

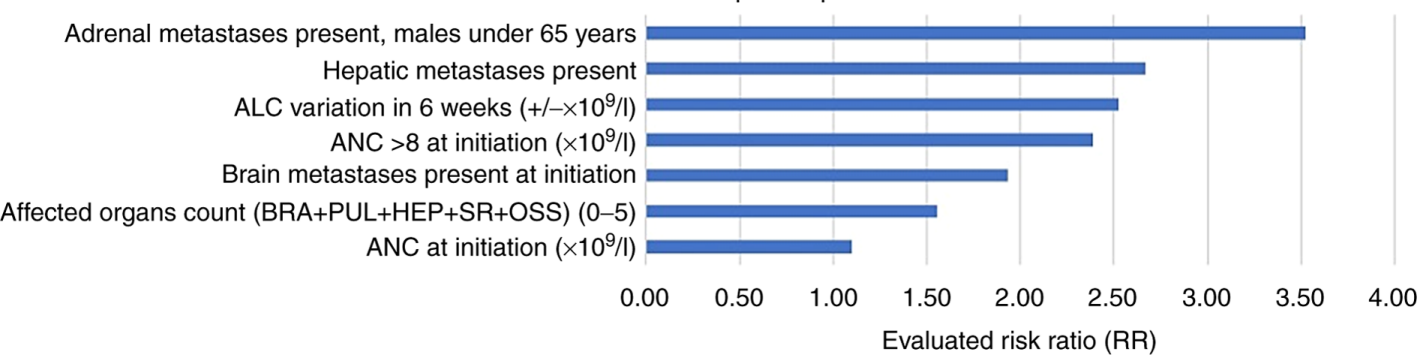

Figure 3. RR for parameters with $\mathrm{P}<0.05$. RR, risk ratio; ALC, absolute lymphocyte count; BRA, brain; PUL, pulmonary; HEP, hepatic; ADR, adrenal; OSS, osseous; ANC, absolute neutrophil count.

Other authors investigated 50 possible predictors of disease-specific survival during nivolumab treatment for NSCLC. Correlations with disease-specific survival were proven for ECOG PS, size of the largest brain metastasis, number of metastatic sites, toxicity, and malignant pleural effusion and correlations with time to treatment failure were confirmed for malignant pleural effusion, number of metastatic sites and number of liver metastases (17).

As lung and breast cancers metastasize to the eye and, although rare, metastatic choroid tumors are the most common type of intraocular malignancy, the patients were screened for associated ocular changes and, if necessary, for treatment options (18-22).

The value of total calcium $<9 \mathrm{mg} / \mathrm{dl}$ (lower normal or hypocalcemia) is shown as a possible negative predictive factor for the duration of nivolumab immunotherapy. It should be noted that hypoalbuminemia is associated with poor immunotherapy results (23) (possibly by increasing the degradation of antibodies) and that hypoalbuminemia is a cause for apparently lower values of total calcium; in conditions of hypoalbuminemia, serum calcium values should be corrected.

Previous studies have shown that before starting nivolumab therapy, $17 \%$ of NSCLC patients present with hypoalbuminemia and $37 \%$ have lost more than $5 \%$ of their weight in the last 6 months. Progression-free survival (PFS) and OS are strongly influenced by albumin levels, hypo- vs. normal albuminemia differences being significant: 5.2 vs. 8.5 months in the case of PFS, respectively 6.9 vs. 18.5 months in the case of OS (23).

Besides the common limitations of a retrospective study, our analysis was hindered by the low number of probands in the subgroups, leading to uncomfortably wide confidence intervals for many investigated variables.
In conclusion, negative predictive factors were identified for the duration of nivolumab treatment: The presence of adrenal metastases (in men under 65 years of age), the presence of liver metastases, neutrophilia at the beginning of treatment (expressed both as ANC and as a value exceeding 8x10 $/ 1$ ), absolute variation (increase) of lymphocytes at 6 weeks of treatment, the presence of brain metastases and the number of metastatic affected organs.

It is important to report early evolutive parameters that are predictive for the total duration of nivolumab treatment as demonstrated for circulating lymphocyte variation in the first 6 weeks.

\section{Acknowledgements}

Professional editing, linguistic and technical assistance was performed by Irina Radu, individual service provider.

\section{Funding}

The present study did not receive specific funding.

\section{Availability of data and materials}

The data generated or analyzed during this study are included in this published article or are available from the corresponding author on reasonable request.

\section{Authors' contributions}

SoS organized the study, analyzed and interpreted the study data and wrote the manuscript. SN, SV, DP, VC, SiS, HF, RD 
and DM analyzed the data and helped to draft the output and critically reviewed the manuscript; $\mathrm{CV}$ interpreted the data and critically reviewed the manuscript for intellectual content. All the authors read and approved the final version of the manuscript for publication.

\section{Ethics approval and consent to participate}

All patients gave their informed consent for the procedure. The study protocol was conducted according to the principles of the Declaration of Helsinki after the approval of the Oncohelp Clinic Ethics Committee (3b/15.09.2020) (Timisoara, Romania). All patients provided written informed consent for the study participation and data collection.

\section{Patient consent for publication}

Not applicable.

\section{Competing interests}

The authors declare that they have no competing interests.

\section{References}

1. Pardoll DM: The blockade of immune checkpoints in cancer immunotherapy. Nat Rev Cancer 12: 252-264, 2012.

2. Niezgoda A, Niezgoda P and Czajkowski R: Novel approaches to treatment of advanced melanoma: A review on targeted therapy and immunotherapy. Biomed Res Int 2015: 851387, 2015.

3. Bansal P, Osman D, Gan GN, Simon GR and Boumber Y: Recent advances in immunotherapy in metastatic NSCLC. Front Oncol 6: 239, 2016.

4. Gill DM, Agarwal N and Vaishampayan U: Evolving treatment paradigm in metastatic renal cell carcinoma. Am Soc Clin Oncol Educ Book 37: 319-329, 2017.

5. Wang C, Thudium KB, Han M, Wang XT, Huang H, Feingersh D, Garcia C, Wu Y, Kuhne M, Srinivasan M, et al: In vitro characterization of the anti-PD-1 antibody nivolumab, BMS-936558, and in vivo toxicology in non-human primates. Cancer Immunol Res 2: 846-856, 2014.

6. Renner A, Burotto M and Rojas C: Immune checkpoint inhibitor dosing: Can we go lower without compromising clinical efficacy? J Glob Oncol 5: 1-5, 2019

7. Agrawal S, Feng Y, Roy A, Kollia G and Lestini B: Nivolumab dose selection: Challenges, opportunities, and lessons learned for cancer immunotherapy. J Immunother Cancer 4: 72, 2016.

8. Yoo SH, Keam B, Kim M, Kim SH, Kim YJ, Kim TM, Kim DW, Lee JS and Heo DS: Low-dose nivolumab can be effective in non-small cell lung cancer: Alternative option for financial toxicity. ESMO Open 3: e000332, 2018.

9. Zhang J, Sanghavi K, Shen J, Zhao X, Feng Y, Statkevich P, Sheng J, Roy A and Zhu L: Population pharmacokinetics of nivolumab in combination with ipilimumab in patients with advanced malignancies. CPT Pharmacometrics Syst Pharmacol 8: 962-970,2019.
10. Cortellini A, Verna L, Porzio G, Bozzetti F, Palumbo P, Masciocchi C, Cannita K, Parisi A, Brocco D, Tinari N and Ficorella C: Predictive value of skeletal muscle mass for immunotherapy with nivolumab in non-small cell lung cancer patients: A 'hypothesis-generator' preliminary report. Thorac Cancer 10: 347-351, 2019.

11. Payne RB, Little AJ, Williams RB and Milner JR: Interpretation of serum calcium in patients with abnormal serum proteins. Br Med J 4: 643-646, 1973.

12. Cusato J, Genova C, Tomasello C, Carrega P, Ottonello S, Pietra G, Mingari MC, Cossu I, Rijavec E, Leggieri A, et al: Influence of vitamin D in advanced non-small cell lung cancer patients treated with nivolumab. Cancers (Basel) 11: 125, 2019.

13. Barta JA, Powell CA and Wisnivesky JP: Global epidemiology of lung cancer. Ann Glob Health 85: 8, 2019.

14. Tamura T, Kurishima K, Nakazawa K, Kagohashi K, Ishikawa H, Satoh $\mathrm{H}$ and Hizawa H: Specific organ metastases and survival in metastatic non-small-cell lung cancer. Mol Clin Oncol 3: 217-221, 2015.

15. Crinò L, Bronte G, Bidoli P, Cravero P, Minenza E, Cortesi E, Garassino MC, Proto C, Cappuzzo F, Grossi F, et al: Nivolumab and brain metastases in patients with advanced non-squamous non-small cell lung cancer. Lung Cancer 129: 35-40, 2019.

16. Karantanos T, Karanika S, Seth B and Gignac G: The absolute lymphocyte count can predict the overall survival of patients with non-small cell lung cancer on nivolumab: A clinical study. Clin Transl Oncol 21: 206-212, 2019.

17. Pantano F, Russano M, Berruti A, Mansueto G, Migliorino MR, Adamo V, Aprile G, Gelibter A, Ficorella C, Falcone A, et al: Prognostic clinical factors in patients affected by non-small-cell lung cancer receiving nivolumab. Expert Opin Biol Ther 20: 319-326, 2020.

18. Preda MA, Popa G, Karancsi OL, Musat O, Popescu SI, Munteanu M and Popa Z: Effectiveness of subconjunctival bevacizumab associated with a laser-based procedure in the treatment of neovascular glaucoma. Farmacia 66: 621-626, 2018.

19. Boruga O, Balasoiu AT, Giuri S, Munteanu M, Stanca HT, Iovanescu G and Preda MA: Caruncular late-onset junctional nevus: Apropos of an anatomo-clinical observation. Rom J Morphol Embryol 58: 1461-1464, 2017.

20. Preda MA, Karancsi OL, Munteanu M and Stanca HT: Clinical outcomes of micropulse transscleral cyclophotocoagulation in refractory glaucoma-18 months follow-up. Lasers Med Sci 35: 1487-1491, 2020.

21. Stanca HT, Munteanu M, Jianu DC, Motoc AGM, Tăbăcaru B, Stanca S, Ungureanu E, Boruga VM and Preda MA: New perspectives in the use of laser diode transscleral cyclophotocoagulation. A prospective single center observational cohort study. Rom J Morphol Embryol 59: 869-872, 2018.

22. Stanca HT, Petrović Z and Munteanu M: Transluminal Nd:YAG laser embolysis-a reasonable method to reperfuse occluded branch retinal arteries. Vojnosanit Preg1 71: 1072-1077, 2014.

23. Lee CS, Devoe CE, Zhu X, Fishbein JS and Seetharamu N: Pretreatment nutritional status and response to checkpoint inhibitors in lung cancer. Lung Cancer Manag 9: LMT31, 2020.

This work is licensed under a Creative Commons Attribution-NonCommercial-NoDerivatives 4.0 International (CC BY-NC-ND 4.0) License. 